Meta

Journal des traducteurs

Translators' Journal

\title{
Terminologie et traduction (cas de l'ornithologie et de la linguistique)
}

\section{Taïeb Baccouche et Salah Mejri}

Volume 45, numéro 3, septembre 2000

La traduction dans le monde arabe

URI : https://id.erudit.org/iderudit/001937ar

DOI : https://doi.org/10.7202/001937ar

Aller au sommaire du numéro

Éditeur(s)

Les Presses de l'Université de Montréal

ISSN

0026-0452 (imprimé)

1492-1421 (numérique)

Découvrir la revue

Citer cet article

Baccouche, T. \& Mejri, S. (2000). Terminologie et traduction (cas de l'ornithologie et de la linguistique). Meta, 45(3), 437-444.

https://doi.org/10.7202/001937ar
Résumé de l'article

Certains voient souvent dans la construction de systèmes terminologiques en L2 un simple habillage en mots de référents ou de concepts bien établis en L1. Or, une telle vision est tellement simplificatrice qu'elle occulte l'extrême complexité de la tâche du terminologue.C'est en partant d'expériences aussi différentes que celles qui couvrent la terminologie ornithologique et la terminologie linguistique en arabe que nous avons essayé de fournir des exemples précis illustrant les grandes difficultés méthodologiques dans ce domaine. 


\title{
Terminologie et traduction (cas de l'ornithologie et de la linguistique)
}

\author{
TAÏEB BACCOUCHE
}

SALAH MEJRI

Université de Tunis I, Tunis, Tunisie

\section{RÉSUMÉ}

Certains voient souvent dans la construction de systèmes terminologiques en $L_{2}$ un simple habillage en mots de référents ou de concepts bien établis en $L_{1}$. Or, une telle vision est tellement simplificatrice qu'elle occulte l'extrême complexité de la tâche du terminologue.

C'est en partant d'expériences aussi différentes que celles qui couvrent la terminologie ornithologique et la terminologie linguistique en arabe que nous avons essayé de fournir des exemples précis illustrant les grandes difficultés méthodologiques dans ce domaine.

\section{ABSTRACT}

Some people often see in the construction of terminological systems in $\mathrm{L}_{2}$ a sort of dressing in words of referents or concepts well established in $L_{1}$. In fact, this point of view is so oversimplified that it conceals the extreme complexity of the terminologist's task.

It is starting from experiences as different as those relating to ornithology and linguistics terminologies in Arabic that we have tried to provide concrete examples to illustrate the great methodological difficulties in this field.

\section{MOTS-CLÉS/KEYWORDS}

terminologie, linguistique, ornithologie, traduction, dénomination

\section{Introduction}

Le traducteur est souvent confronté à des difficultés liées aux différences intrinsèques entre les systèmes linguistiques en présence dans l'acte de traduire. Il a nécessairement recours devant ces difficultés à l'une des deux solutions suivantes: la recherche de correspondants ou, à défaut, l'emploi d'équivalents (cf. Lederer 1994).

Il est des domaines où de telles solutions ne sont pas en relation complémentaire; ce sont ceux qui se distinguent par un appareil conceptuel clairement défini, ce qui s'illustre aisément par des domaines techniques et scientifiques. La traduction des textes de description des différents domaines et théories impose au traducteur la recherche d'un correspondant à tout concept bien délimité dans la langue de départ.

D'aucuns y verraient la proposition d'un simple signifiant, puisque le concept représente la matière même du texte traduit; en fait, il s'agit d'une opération beaucoup plus complexe qui implique à la fois les systèmes linguistiques d'arrivée dans toutes leurs dimensions (surtout sémantique), les descriptions qui y sont disponibles sur les questions étudiées et, par conséquent, tout ce qui en découle comme complications nécessitant des solutions adéquates.

Nous illustrons dans ce qui suit cette problématique par des exemples empruntés à la terminologie ornithologique et linguistique, deux domaines qui s'opposent 
par la nature de leur objet de recherche et de leur dénomination, et par la nature des problèmes théoriques qu'ils posent.

\section{La terminologie ornithologique}

Il s'agit d'un projet de recherche national financé par l'ancienne Fondation nationale pour la recherche, regroupant des linguistes et des ornithologues (années 19911992), dont l'objectif était de doter la langue arabe d'une terminologie dénommant toutes les espèces d'oiseaux vivant en Tunisie et tout ce qui est en rapport avec leur mode de vie, leur habitat, leur environnement, etc.

Ce qui distingue ce domaine, c'est la présence d'un référent précis jouissant d'une description scientifique disponible en anglais et en français avec toutes les illustrations iconiques qui aident à distinguer les espèces les unes des autres. On serait donc tenté a priori de forger des termes estimés correspondants à ceux de la terminologie bien établie dans les langues de départ.

Cette solution, bien que pratiquée assez souvent, ne tiendrait pas compte de la complexité des données linguistiques tant dans les langues de départ que dans les langues d'arrivée, ce qui réduirait l'opération de traduction à une simple transposition de termes, ignorant ainsi l'économie des systèmes en présence, les rapports que les termes disponibles en $\mathrm{L}_{1}$ entretiennent avec la nomenclature latine d'une part et avec le lexique disponible dans le langage courant avec ses diverses variantes d'autre part, ainsi que les différentes valeurs dont ces dénominations sont investies.

En arabe, même si on trouve des données de base similaires, il y a lieu de mentionner l'importance des cases vides et du clivage littéral/dialectal.

La tâche du traducteur terminologue se trouve ainsi conditionnée par un certain nombre d'exigences méthodologiques dont nous retenons:

- la nécessité d'une enquête de terrain dont l'objectif est de collecter les dénominations locales en vigueur, d'une recherche lexicographique et de l'analyse de textes spécialisés ou autres visant la détermination de la terminologie en cours dans le littéral;

- l'évaluation d'une telle terminologie en vue d'en dégager les insuffisances, les contradictions, les lacunes afin de bien délimiter les données exploitables dans la traduction;

- le choix d'un ensemble de repères linguistiques déterminant la configuration des dénominations proposées en $\mathrm{L}_{2}$ : mot simple/mot composé, recours au calque, l'exploitation des données dialectales, transfert référentiel, exploitation du patrimoine, etc.;

- le traitement des dénominations par séries, jouant ainsi sur les différents paradigmes linguistiques en correspondance avec l'organisation référentielle des données, et abandonnant le traitement par termes isolés;

- le caractère déterminant du contenu des descriptions scientifiques dans l'élaboration de la dénomination proposée : habitat, mode de vie, morphologie, nidification, nourriture, etc.

Partant de cet ensemble de repères méthodologiques, il nous a été possible de résoudre un grand nombre de problèmes impliquant les données de base indiquées dont nous fournissons à titre d'illustration quelques exemples:

a. le calque

Bien que l'emprunt soit pleinement justifié dans le cas où on ne dispose pas de dénomination arabe, surtout pour certains oiseaux migrateurs, nous n'avons pas éprouvé 
le besoin d'y recourir parce que la matière lexicale disponible est suffisamment abondante. Cependant, nous avons opté dans quelques cas pour le calque. Cette solution s'avère très économique dans les situations où il $\mathrm{y}$ a correspondance entre les déterminés et leurs déterminants dans $\mathrm{L}_{1}$ et $\mathrm{L}_{2}$ :

$$
\begin{aligned}
& \text { oie cendrée [wazza rama:dijja] }
\end{aligned}
$$

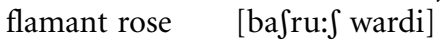

$$
\begin{aligned}
& \text { aigle botté [\%uqa:b musarwal] }
\end{aligned}
$$

\section{b. l'exploitation des dénominations dialectales}

Vu la hiérarchie établie entre le littéral et le dialectal, une telle solution semble inadéquate. Or, nous pensons qu'une terminologie, si elle cherche à être efficace, ne doit pas ignorer le lexique disponible même s'il est dialectal. Ce choix consacre en fait l'une des sources de création lexicale en arabe standard, où s'intègre facilement un très grand nombre de mots d'origine dialectale passant le plus souvent à travers le discours journalistique ou même littéraire. Les exemples retenus figurent déjà parmi les dénominations recueillies pendant les enquêtes dans les régions:
bécasse
[bu:xlal]
échasse

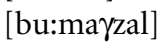

\section{c. la structure binaire des dénominations}

Vu la diversité des genres et des espèces, seules les dénominations complexes fournissent un outil linguistique assurant la transparence des termes forgés, raison pour laquelle nous avons systématiquement opté, à l'instar des dénominations en $\mathrm{L}_{1}$, pour la structure binaire «Déterminé + Déterminant».

Le déterminé, le plus souvent un terme consacré d'une manière ou d'une autre dans la langue pour servir de générique renvoyant aux divers oiseaux appartenant au même genre, est choisi en fonction à la fois du déterminé en $\mathrm{L}_{1}$, des termes disponibles en $\mathrm{L}_{2}$ dénommant espèces, genres, familles et classes, et des termes locaux colligés pendant les enquêtes. Le déterminant, qui est soit un terme adjectival soit un nom annexé, assure une fonction distinctive dans les sous-espèces en renvoyant à des caractéristiques tirées des descriptions relatives à la morphologie, au mode de vie, au milieu, à la nidification, etc., créant ainsi un paradigme ouvert où pourraient s'intégrer les nouvelles dénominations non encore établies. Ce choix est d'autant plus justifié que ce projet couvrira à une étape ultérieure toute la région arabe. Dans les cas de saturation, on construit des dénominations ternaires; solution qui demeure relativement rare :

$$
\begin{array}{ll}
\text { alouette calandre } & \text { [qubbaratuzzar:i] } \\
\text { alouette de Clot-Bey } & \text { [qubbaratulba:ji] } \\
\text { alouette lulu } & \text { [qubbaratulya:bi] } \\
\text { alouette des champs } & \text { [qubbaratulhuqu:li] }
\end{array}
$$

Il arrive que la variation dans les sous-espèces soit traduite aussi par un jeu sur le déterminé. Ainsi en est-il de ces espèces d'alouettes qui se distinguent des autres par leur petite taille: au lieu d'employer le terme générique [qubbara], on lui substitue le diminutif. 
alouette calandrelle [qubbajratuzzar:i]

choisissant ainsi de jouer sur le déterminé non sur le déterminant comme c'est le cas en $\mathrm{L}_{1}$.

\section{d. la néologie comme mode de formation terminologique}

Il est des cas où le lexique disponible ne rend pas compte parfaitement des spécificités du genre ou de l'espèce. La solution la plus adéquate pour s'assurer de la transparence nécessaire demeure la création d'un néologisme qui est en fait un mot du lexique auquel on assigne une fonction dénominative, et ce, en rapport avec certaines spécificités: nous avons attribué par exemple à la cisticole des joncs le terme [mawwa:3ullasal] dont le déterminé évoque la manière onduleuse de voler; le terme choisi pour le bouvreuil githagine, [zarasi:], se justifie par son chant qui ressemble à une sonnette électrique et à un klaxon. La néologie réside donc dans le lien établi entre le référent et le mot choisi dans le stock lexical non dans la création ex nihilo.

\section{e. le traitement par série}

Du moment que le traitement par terme favorise la multiplicité des solutions et risque d'être une source de confusion, seul le traitement par série semble être l'outil paradigmatique adéquat. Ce qui précède laisserait entendre que nous avions suivi dans la traduction les séries de $\mathrm{L}_{1}$. Or, il s'avère que les dénominations dans la même série de $\mathrm{L}_{1}$ ne recoupent pas les séries dans les dénominations latines. Les transposer telles quelles en arabe priverait nos propositions de la cohérence exigée par le système, raison pour laquelle nous avons créé de nouvelles séries fondées sur les caractéristiques contenues dans les descriptions scientifiques. Le terme [ $\div$ uqa:b] couvre à la fois les aigles, le balbuzard et la circaète; celui de [nisr] employé dans l'usage courant pour désigner l'aigle, nous l'avons assigné au vautour qui appartient à la famille des accipitridés.

Au terme de ces exemples de traitement terminologique ornithologique, il apparaît clairement que le référent, avec tout ce qu'il implique comme données objectives, reste un élément déterminant dans les choix établis. C'est en exploitant les indications fournies sur les familles, les genres et les espèces que le choix linguistique peut assurer la transparence recherchée et que les contradictions relevées en $\mathrm{L}_{1}$ et en $\mathrm{L}_{2}$ sont surmontées.

Cet outil nous fait défaut dans le cas de la terminologie linguistique où seuls le concept et la notion assurent la même fonction.

\section{La terminologie linguistique}

Les données de base dans le domaine linguistique obéissent à une logique différente. $\mathrm{Si}$, dans le domaine ornithologique, les référents, échappant à toute appréciation subjective, sont mal ou non dénommés en $\mathrm{L}_{2}$, dans celui de la terminologie linguistique, outre l'aspect référentiel qui ne se présente pas de la même manière, il y a lieu de constater qu'il existe en $\mathrm{L}_{1}$ et $\mathrm{L}_{2}$ une grande complexité terminologique de départ: en $\mathrm{L}_{1}$, la terminologie linguistique hérite d'une terminologie grammaticale qu'elle reprend et enrichit par une grande diversité terminologique favorisée par l'apport théorique des écoles et des approches renouvelées; $\mathrm{en}_{2}$, se greffent à la terminologie 
grammaticale des terminologies de linguistique générale et appliquée instables, parce que pour la plupart individuelles et non consacrées.

Cette situation se complique davantage par le fait que chaque terminologie reflète un système linguistique spécifique et des approches différentes: en $\mathrm{L}_{1}$, il s'agit d'une langue indo-européenne dont les descriptions grammaticales sont empreintes de la tradition gréco-latine; par contre, en $\mathrm{L}_{2}$, on a affaire à une langue sémitique dont la tradition grammaticale millénaire véhicule une vision de la langue fortement imprégnée par des considérations religieuses ${ }^{1}$.

Devant la complexité de cette situation, l'effort terminologique a pris la forme de glossaires, d'index et de publications variées (traductions, thèses, articles, etc.) qui fournissent pour la plupart des listings en $\mathrm{L}_{1}$ suivis de termes arabes, ce qui a donné lieu à une grande confusion dont nous avons essayé de rendre compte à propos des dénominations basiques relatives aux différentes configurations que peut prendre le langage naturel (langue, parole, parler, idiome, lecte, idiolecte, dialecte, vernaculaire, argot, jargon, patois, sabir, pidgin, créole; pour les détails, cf. Baccouche et Mejri 1996). Trois insuffisances fondamentales se dégagent de ces pratiques: les terminologies sont souvent établies indépendamment de leurs supports textuels d'origine et, par voie de conséquence, de leurs contenus conceptuels précis; au contraire, elles sont réduites à des inventaires de termes pris isolément et auxquels sont proposés des correspondants arabes. Le résultat, c'est une situation d'anarchie où les contours des notions sont flous, où certains concepts sont pris pour d'autres et où coexistent à la fois la profusion et la pénurie terminologiques.

Pour pallier de telles insuffisances, nous avons tenté d'appliquer dans notre pratique les principes méthodologiques suivants:

1. Convaincus que l'élaboration de la terminologie d'un domaine tel que la linguistique ne peut se faire indépendamment des textes d'origine qui en explicitent les contenus, en précisent les contours, en déterminent les valeurs par rapport aux autres termes du système et en fournissent des illustrations claires, nous pensons que l'élaboration, par exemple, d'un dictionnaire de linguistique capable de participer efficacement à la normalisation de la terminologie linguistique en arabe passe nécessairement par la traduction des textes fondateurs (cf. Guermadi 1966; Baccouche 1981; Guermadi, Agina et Chaouch 1985; Baccouche et Mejri 1993 et 2000).

2. Ce premier principe conditionne la qualité des choix opérés, en ceci qu'il permet de distinguer les concepts de linguistique générale de ceux qui sont propres aux systèmes linguistiques particuliers. Dans le premier cas, la tâche est relativement aisée. C'est ce qui explique le consensus actuel relevé, en Tunisie en particulier, malgré la grande profusion terminologique constatée au départ. Dans la production linguistique, des termes comme [dali:lun] «signe», [da:llun] «signifiant», [madlu:lun] «signifié», [dla:latun] «signification», [sawtamun] «phonème», [harfamun] «gra-

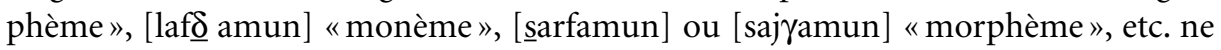
posent pas de problèmes particuliers d'emploi et de compréhension.

La situation est beaucoup plus compliquée quand il s'agit de traduire des faits relatifs à une langue donnée. Les problèmes évoqués représentent un écueil certain, et par conséquent toute solution exige les précautions méthodologiques suivantes:

- éviter systématiquement les transferts automatiques: Toute notion doit être bien située dans son cadre et bien illustrée par des faits linguistiques qui aident à en délimiter les contours avec le maximum de précision. Décrire par exemple la morphologie lexicale 
arabe en termes de dérivation et de composition, c'est d'une certaine manière déformer les données de base du système morphologique arabe qui repose sur deux concepts descriptifs fondamentaux, celui de racine consonantique et celui de schème: la racine $[\mathrm{KTB}]$ épouse plusieurs schèmes selon qu'on veut exprimer un procès accompli $\left(c_{1}\right.$ a $c_{2}$ a $c_{3}$ a) [kataba] ou inaccompli $\left(j\right.$ a $\left.c_{1} c_{2} u c_{3} u\right)$ [jaktubu], un nom d'agent $\left(c_{1}\right.$ a: $c_{2}$ i $c_{3}$ $\mathrm{u}$ n) [ka:tibun], un nom de patient ( $\mathrm{m} \mathrm{a}_{1} \mathrm{c}_{2} \mathrm{u}: \mathrm{c}_{3} \mathrm{u}$ n) [maktu:bun], etc.

Cette caractéristique morphologique est importante au point de faire de l'arabe un système d'une très grande régularité. ${ }^{2}$

- être conscient de la très grande difficulté du transfert terminologique d'un système vers un autre, car raison en est que les concepts ne peuvent être appréhendés isolément. L'exemple des fonctions de l'adjectif en français (épithète, attribut du sujet ou de l'objet et apposition) illustre bien note propos. Les trois fonctions présupposent une répartition en parties du discours qui n'est pas la même en français et en arabe par exemple (neuf en français et trois seulement en arabe: nom, verbe et particule), un paradigme de fonctions qui sont spécifiques aux parties du discours ou qui les transcendent. Les termes arabes qui sont susceptibles de leur servir de correspondants, étant eux-mêmes intégrés dans un autre système, ne sont pas censés couvrir les mêmes réalités. Si on en choisit un terme, il est recommandé d'en voir de très près le champ d'action. L'exemple de l'attribut dans les phrases suivantes:

Le local est agréable.

Je trouve ce local agréable.

Je cherche un local agréable.

correspond dans les phrases arabes équivalentes à trois fonctions différentes: [xabar], l'équivalent du prédicat; [ha:l], l'équivalent approximatif du complément de manière; [na:t], l'équivalent de l'épithète ${ }^{3}$.

C'est dire la complexité de la tâche! En prendre conscience éviterait les solutions hâtives qui ne font que rendre les solutions adéquates encore plus difficiles à atteindre. être attentif aux confusions conceptuelles: les précautions déjà mentionnées ne suffisent pas à faire éviter certaines confusions conceptuelles quand les termes consacrés dans $\mathrm{L}_{1}$ et $\mathrm{L}_{2}$ ne sont pas suffisamment étudiés dans leurs structurations conceptuelles. Quoi de plus évident que les notions syntaxiques de phrase nominale et de phrase verbale si couramment employées! Pourtant, elles ne renvoient pas aux mêmes contenus ni aux mêmes réalités linguistiques en français et en arabe. Si l'opposition nominale/verbale en français est fondée sur l'absence ou la présence d'un verbe dans la structure de la phrase, elle repose en arabe sur la présence du nom ou du verbe en tête de phrase. Parler d'une phrase nominale en arabe ne signifie pas qu'elle ne comporte pas de verbe. Le rapprochement entre les deux systèmes montre que la structure de certaines phrases nominales arabes est identique à celle de la phrase verbale française :

$\mathrm{SN}-\mathrm{SV}$

L'enfant / joue au ballon

[?at tiflu / jal:abu bilkurati]

Les termes sont donc disponibles dans ce cas en $\mathrm{L}_{1}$ et $\mathrm{L}_{2}$ mais ne renvoient pas aux mêmes contenus conceptuels.

- éviter les pièges des faux amis terminologiques: en phonétique et en phonologie, il est courant de traduire l'assimilation par [idga:m], terme emprunté à la terminologie grammaticale arabe et relatif au phénomène articulatoire qui consiste à prononcer sous la forme d'une géminée deux consonnes successives identiques ou non; cette articulation exige, dans le cas de la non-identité des consonnes, une phase d'assimilation facilitant le redoublement. L'assimilation est un phénomène combinatoire qui peut être total ou partiel, progressif ou régressif, mais exigeant dans tous les cas de figure une différence entre les phonèmes de départ et la présence de traits en commun à l'arrivée.

Pour marquer la différence entre les deux phénomènes, certains linguistes arabes 
modernes traduisent assimilation par [tama:qul] («identité»), tombant ainsi dans le piège inverse puisqu'ils mettent ainsi en saillance un seul aspect du phénomène, l'identité.

- s'assurer qu'un minimum de transparence soit garantie dans les propositions avancées. Pour ce faire, nous considérons que, tout comme pour l'ornithologie, la référence conceptuelle représente l'élément le plus déterminant. Ainsi, pour rendre compte de l'anaphore, pour laquelle on ne dispose pas de terme consacré en arabe, le terme [tarda:d] nous semble traduire les fonctions anaphoriques essentielles que sont la reprise et la redondance, sèmes véhiculés par le terme arabe proposé.

\section{Conclusion}

Considérer que l'établissement d'un appareil terminologique quelconque est une simple opération de confection de termes en $\mathrm{L}_{2}$, c'est réduire cette opération, qui est d'une complexité extrême, à un simple habillage en mots et occulter par là tous les problèmes linguistiques et conceptuels que cela implique.

$\mathrm{Ne}$ pas être conscient de la dimension épistémologique de ce travail donnerait lieu à une maîtrise partielle, confuse et désordonnée des sciences et des techniques étudiées à travers une terminologie approximative.

Notre objectif dans ce travail, conçu à partir d'expériences aussi différentes que riches en enseignements, n'est pas de fournir des recettes qui, dénuées de tout fondement théorique, ne seraient d'aucun recours, mais d'attirer l'attention sur des repères méthodologiques qui sont de nature à mettre en relief l'extrême complexité de la création terminologique dans une situation de traduction.

\section{NOTES}

1. Dans la tradition grammaticale arabe, l'interprétation du texte sacré qu'est le Coran était au centre de la réflexion linguistique, ce qui a occulté longtemps la vision évolutive de la langue.

2. Ce qui permet entre autres la lecture de textes arabes non voyellés.

3. On peut encore citer les notions de passif, de mode, de voix, etc.

\section{RÉFÉRENCES}

(Note: Pour les références terminologiques arabes, nous renvoyons à Baccouche et Mejri 1996.) BAccouche, T. (1994) : L'emprunt en arabe moderne, Tunis, Beït Al-Hikma - Carthage et IBLV, Université de Tunis I.

Baccouche, T. et S. Mejri (1993): Du mot, Tunisie, Dar El Janoub (en arabe).

Baccouche, T. et Salah M. (1996): «De la problématique de l'appareil terminologique linguistique arabe (spécimen méthodologique) », Études linguistiques, 1, Tunisie, Association Tunisienne de Linguistique (en arabe).

Borsson, C. et P. Thoiron (1997): Autour de la dénomination, Lyon, Presses universitaires de Lyon.

Meta, 41-4 (numéro spécial «Dénomination»), Montréal, Presses de l’Université de Montréal. Felber, H. (1984) : Terminology manual, Paris, UNESCO.

Heinzel, H., R. Fitter et J. Parslow (1985): Oiseaux d'Europe, d'Afrique du Nord et du MoyenOrient, Neuchâtel, Delachaux et Niestlé.

Larose, R. (1989): Théories contemporaines de la traduction, $2^{\text {e }}$ éd., Montréal, Presses de l'Université du Québec.

Lederer, M. (1994): La traduction aujourd'hui, Paris, Hachette. 
Messaoudi, L. (1998): "Technolectes bilingues (français-arabe) et mode de dénomination", Revue Tunisienne des Sciences sociales, $\mathrm{n}^{\circ}$ 117, Actes du colloque La mémoire des mots (Taïeb Baccouche, Salah Mejri et André Clas, dir.), Tunis, CERES.

MejRi, S. (1995): La néologie lexicale, La Manouba, Publications de la Faculté des Lettres.

- (1997): Le figement lexical. Descriptions linguistiques et structuration sémantique, La Manouba, Publications de la Faculté des lettres.

— (1998): «La conceptualisation dans les séquences figées", L'information grammaticale, numéro spécial «Tunisie», mai 1998, p. 41-48.

— (2000): «Figement et dénomination», Meta, 45-4, Montréal, Presses de l'Université de Montréal.

Mejri, S., G. Gross, T. Baccouche et A. Clas (1998): Le figement lexical, Actes de la $1^{\text {re }}$ R.L.M, Tunis, CERES.

Pergnier, M. (1993): Les fondements socio-linguistiques de la traduction, Lille, Presses universitaires de Lille.

RondeAu, G. (1984): Introduction à la terminologie, Chicoutimi, Gaëtan Morin. 